\title{
Acute Pneumonia in Children: The Price of Illusions and Delusions
}

Received: January 11, 2017; Accepted: January 30, 2017 ; Published: February 05, 2017

\section{Introduction}

"Pneumonia was described 2,500 years ago, by Hippocrates, the father of medicine". To date, medical science has a broad view of the role of lungs in the body, including their non-respiratory functions. The huge volume of scientific information gives an idea of the dynamics of changes in the body of patients with pneumonia and finds an explanation for the ineffectiveness of treatment and the causes of complications. However, the evaluation of the current situation in this section of clinical medicine looks hopeless, and her improvement is very uncertain.

"Pneumonia is a leading cause of hospitalization among children in the USA, with medical costs estimated at almost \$1 billion in 2009. Despite this large burden of disease, critical gaps remain in our knowledge about pneumonia in children.

"The rates of parapneumonic effusion have been increasing in the USA and Europe over recent years, and it is now encountered in approximately $40 \%$ of all patients with bacterial pneumonias" (Thorax 2011; 66: 815e822. doi:10.1136/thx.2010.142604).

"Pediatric pleural empyema has increased substantially over the past 20 years and reasons for this rise remain not fully explained." (Pediatric Pulmonology 50: 721-726 (2015).

"Pneumonia puts thousands of young children in the hospital each year at a cost in the USA. of about $\$ 1$ billion, not to mention suffering of kids and hardship for their families."

These quotations reflect the current state of the problem under the world's best health systems. These results are quite logical: For many years, all the ideas, efforts and resources are directed, as a rule, to combat pathogens of acute pneumonia (AP).

At the beginning of my medical career, I took a time when all purulent or destructive complications of AP attributed only a staphylococcus infection. When Staphylococcus aureus began to lose the leading position, some researchers rushed to declare victory over him. The final outcome of this confrontation is known: the "winners" received a trophy MRSA, Staphylococcus has retained its place in the etiology of AP and its purulent complications, and MRSA is often detected in healthy humans as a "shocking" representative of symbiotic microflora.

Following the dominant "bogey" in the etiology of AP proved Streptococcus pneumoniae. In this situation, the antibacterial

\section{Igor klepikov}

Dana Children's Hospital, Sourasky Medical Center, Tel Aviv Sourasky Medical Center, Russia

Corresponding author: Igor klepikov

igor.klepikov@yahoo.com

Dana Children's Hospital, Sourasky Medical Center, Tel Aviv Sourasky Medical Center, Russia.

Tel: $+972-3-697-3426$

Citation: klepikov I. Acute Pneumonia in Children: The Price of Illusions and Delusions. J Pediatr Care. 2017, 3:1.

medical assistance was supplemented by total vaccination of the population in developed countries. The results of this campaign also fell short of expectations. "Among children $\leq 18$ years of age, the annual empyema-associated hospitalization rates increased almost 70\% between 1997 and 2006, despite decreases in the bacterial pneumonia and invasive pneumococcal disease rates. Pneumococcal conjugate vaccine is not decreasing the incidence of empyema". "Available data show that pneumococcal bacteria are resistant to one or more antibiotics in $30 \%$ of cases".

Currently, the image of "new" infectious threats arises. "Respiratory viruses, rather than bacterial pathogens, were most commonly detected in children hospitalized with pneumonia". "This ground-breaking study shows how badly we need faster, less-expensive diagnostic tests for doctors to accurately diagnose the cause of pneumonia so they can effectively treat it." The results help define the role of viruses as major players in Pediatric pneumonia and shows a need for new therapies that can reduce the severity of viral pneumonia," says Chris Stockmann, co-investigator and senior research analyst at the University of Utah." Among children diagnosed with pneumonia, viral infections were much more common than bacterial infections (73 vs. 15 percent), and respiratory syncytial virus (RSV) was the most commonly detected pathogen". It is significant that the frequency of detection of these viruses in healthy people is not statistically different from their etiological role in acute pneumonia.

Suggestions for improving the etiological diagnostics and etiotropic treatment are merely forced a tactical step. A 
radical solution to this problem cannot be achieved only by tactical restructurings. Such changes occurred periodically over the years and their outcomes are listed above. Firstly, it is necessary to remember that AP is not contagious infectious disease and its causative agents are representatives of symbiotic microflora. Secondly, it is well known that even the coincidence of pathogens (for example- with sore throat or skin inflammation) AP will differ significantly in character and severity of their clinical manifestations. Acute inflammatory process in the field of pulmonary circulation inevitably violates its coordination with the system blood supply. Individual manifestation of the disease depends, on the one hand, the speed of development of the inflammatory reaction and subsequent shifts of homeostasis, and, on the other hand, adaptive capacity.

For a real solution to this problem, we need to change its strategy and existing concepts of the pathogenesis of the disease. This is the part of my knowledge and experiences that await its wide implementation. 